\title{
High Signal Resolution Pulse Oximetry as a Prognostic Indicator of Radiotherapy Toxicity: A Pilot Study
}

\author{
Karolina Jezierska ${ }^{a}$ Agnieszka Macała ${ }^{b}$ Ryszard Krzyminiewskic \\ Piotr Woźniak $^{\mathrm{b}}{\text { Magdalena } \text { Łukowiak }^{\mathrm{b}} \text { Anna Sękowska-Namiotko }}^{\mathrm{d}}$ \\ Wojciech Podraza ${ }^{a}$
}

${ }^{a}$ Department of Medical Physics, Pomeranian Medical University, Szczecin, Poland; ${ }^{b}$ Department of Medical Physics, West Pomeranian Oncology Center, Szczecin, Poland; 'Medical Physics Division, Adam Mickiewicz University,

Poznań, Poland; 'Department of Metrology and Optoelectronics, Gdańsk University of Technology, Gdańsk, Poland

\section{Keywords}

Cardiotoxicity of radiotherapy $\cdot$ Pulse oximetry $\cdot$ Breast and lung cancers · Pulse wave analysis

\begin{abstract}
Introduction: Radiotherapy plays a prominent role in the multidisciplinary treatment of cancers. Despite new irradiation techniques, radiation-induced cardiotoxicity remains a leading cause of morbidity and mortality. Therefore, prognostic indicators of radiotherapy toxicity are essential. This study aimed to investigate the influence of applied radiotherapy on the pulse wave recording and thereby on cardiovascular health, as well as to assess whether high signal resolution (HSR) pulse oximetry could be used as a prognostic indicator of radiotherapy toxicity. Methods: A total of 19 patients treated with radiotherapy to the lung or left breast were analyzed. Pulse oximetry was performed on all eligible patients (before and after the administration of a radiation dose) using the PULS-HSR program, which increases the resolution of the pulse wave recording. Results: Among the an-
\end{abstract} target, as well as the treatment duration, significantly positively correlated with the change in ventricle/aorta volume ratio. The ventricle/aorta volume ratio parameter is the ratio between the area under the HSR pulse wave generated by the contraction of the left ventricle until the aortic valve closes and the area of the HSR pulse wave responsible for expansion and contraction of the aorta. Conclusion: The pilot study indicates HSR pulse oximetry, especially the ventricle/aorta volume ratio parameter, as a potential prognostic indicator of toxicity from radiation for breast and lung cancers.

(c) 2021 S. Karger AG, Basel

\section{Introduction}

Breast cancer is the most commonly occurring cancer in Polish women (17,142 cases per year), characterized by high curability rates and a 5 -year survival rate of $90 \%$ [1]. In men, lung cancer is the most frequently diagnosed cancer worldwide and the leading cause of cancer-related

Correspondence to:

Karolina Jezierska, karo@ pum.edu.pl 
death (1.4 million deaths/year, 18\%). In Poland, there are $>21,000$ new cases of lung cancer each year, accounting for over 22,000 deaths (data from the Polish National Cancer Registry, 2013).

Radiotherapy plays a major role in the multidisciplinary treatment of both breast and lung cancers. New radiotherapy techniques, such as the deep inspiration breath hold or respiratory gating, have significantly reduced the radiation dose received by the heart during radiotherapy. For example, during breast cancer treatment, the heart will receive, on average, a radiation dose of 1-5 Gy [2-8]. However, as the risk of myocardial infarction or death due to ischemic disease increases by $7.4 \%$ per Gy of radiation administered to the heart, without any apparent threshold below which toxicity is not observed, radiation-induced cardiotoxicity remains a leading cause of morbidity and mortality $[9,10]$. Furthermore, cardiotoxicity can be found even $>20$ years after finishing causal treatment. Therefore, monitoring early and late reactions to radiotherapy is key for improving long-term treatment outcomes. Ideally, clinicians should develop treatment plans that minimize the absorbed dose to the heart while maintaining locoregional disease control at the highest possible level. In this respect, finding new prognostic indicators of radiotoxicity will facilitate the clinical decision-making process.

- Pulse oximetry is a simple, effective, painless, and noninvasive method for the spectrophotometric measurement of oxygen saturation of arterial blood $\left(\mathrm{SpO}_{2}\right)$, with simultaneous heart rate registration. By measuring changes in red and infrared light absorbances over time, the saturation level can be determined due to the different absorption spectra of deoxy- and oxyhemoglobin [11]. High signal resolution pulse oximetry (or high signal resolution pulse wave [HSR-PW]) uses the method of linear transformation based on Fourier analysis to provide increased resolution [12, 13]. Pulse wave numerical analysis enables a series of parameters characterizing the cardiovascular system to be determined, including of course heart rate (bpm) and saturation (\%) but also [14] the following:

- Ventricle/aorta volume ratio - the indicator is calculated as the ratio of area under the HSR pulse wave generated by the left ventricle until the aortic valve closure to the area of the pulse wave responsible for expanding and shrinking the aorta. It indicates aortic compliance on changes in blood pressure caused by contraction of the left ventricle.

- Aorta valve - the indicator is calculated as the ratio of pulse wave HSR on the closure of the aortic valve to the maximum pulse wave amplitude left ventricle.
- Pulsatility index - the indicator is calculated as in the Doppler study, which is the ratio of the difference between maximum-minimum amplitude of the pulse wave HSR left ventricle to the average pulse wave evolution of a single heart.

- $k 2$ index - the amplitude ratio of peak 1 to peak 2 on the HSR pulse curve. It informs about possible abnormalities of the aortic and aortic valve function.

- Artery dynamic - indicator which is the sum of the amplitudes of the wave pulse HSR values below and above the average. It tells about flexibility and cardiovascular vulnerability in the way chamber-finger.

These parameters not only provide information about the pulse and saturation but also about the patient's arteries and heart. Therefore, HSR-PW is a useful diagnostic tool in cardiology [15].

In this pilot study, we investigated the influence of applied radiotherapy on the pulse wave recording, as an indicator of cardiovascular health. We aimed to determine whether HSR pulse oximetry could be used as a prognostic indicator of radiotherapy toxicity. Since the ionizing radiation absorbed by the heart muscle affects its work of the heart chambers and the valves, changes in some parameters of the pulse wave may probably be related to the absorbed dose. Information which we can get from a pulse wave is related to the condition of patient's arteries and heart [14-16].

\section{Materials and Methods}

The study included 19 patients irradiated for left-sided breast cancer (13 patients) and lung cancer (6 patients) in the West Pomeranian Oncology Centre in Szczecin. Conformal radiotherapy at an X-ray energy of $6 \mathrm{MeV}$ was used for both cancers; the deep inspiration breath hold technique was used in the case of breast irradiation.

Correlations between the radiation dose and pulse wave parameters were examined. An example screenshot showing the HSR pulse oximetry results is shown in Figure 1. The standards were established on the basis of studies of healthy people in various age groups. Therefore, the analyzed treatment duration varied among the patients and did not always cover the whole duration of treatment.

The test was performed with the patient in the supine position, just before and just after the radiotherapy treatment. A pulse oximeter CMS-50Ew was attached to the patient's finger on the left hand and used to register the pulse wave. The test lasted $1 \mathrm{~min}$ in duration. Recordings of pulse oximetry data were analyzed with the PULS-HSR program, which increases the resolution of the pulse wave recording. Since the pulse oximetry curve directly measures changes in blood volume at the measurement site, that is, on the finger, the area under the curve is a direct reflection of changes in blood volume (Fig. 2).
Jezierska et al. 


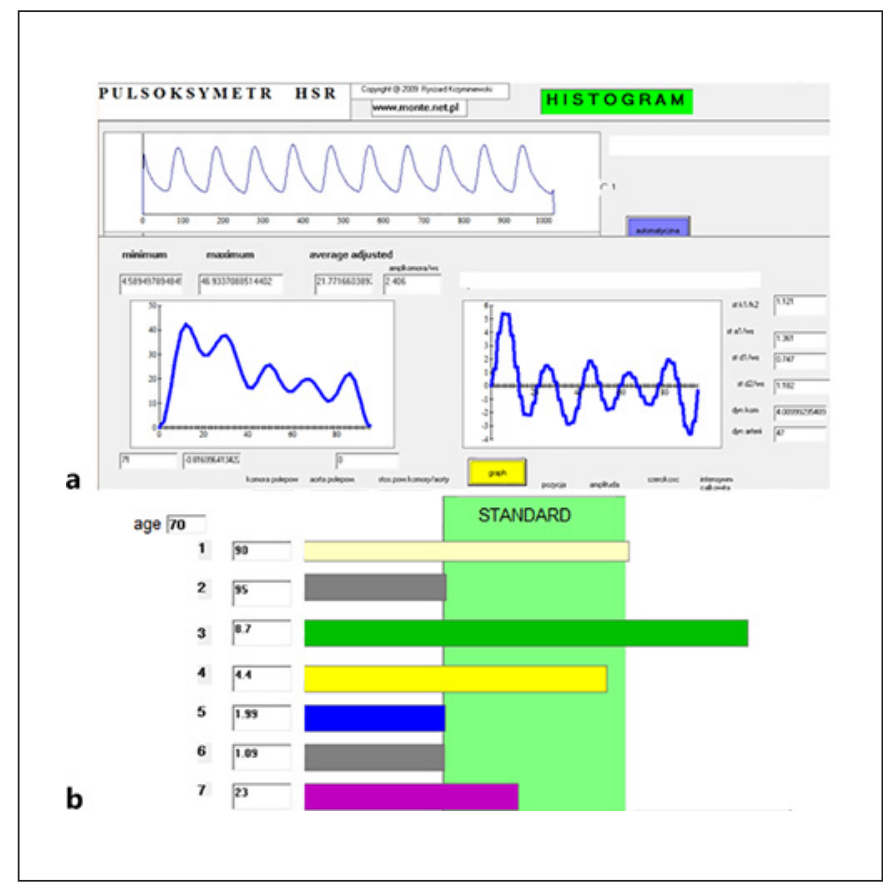

Fig. 1. An example screenshot showing the HSR pulse oximetry results. a The pulse wave recording. $\mathbf{b}$ Bar graph presentation of the data (age-specific reference ranges are marked in green). $\mathrm{Pa}$ rameters: 1 , pulse; 2 , saturation; 3 , ventricle/aorta volume ratio; 4 , aorta valve; 5 , pulsatility index; $6, k 1 / k 2$ index; 7 , artery dynamic. HSR, high signal resolution.

The procedure for enhancement of signal resolution by the linear transformation method was described in previous studies [14]. It is based on the calculation described as a plot of 2 functions:

$$
\mathrm{F}(x)=\int \mathrm{K}\left(x-x^{\prime}\right) \psi\left(x^{\prime}\right) \mathrm{dx} x^{\prime},
$$

where the function $\psi\left(x^{\prime}\right)$ describes the positions and intensities of individual lines and $K\left(x-x^{\prime}\right)$ is a function (core) describing the shape of an individual line. In our calculations, we chose the Gaussian line as a core. To find the positions and intensities of the lines, the explicit form of the function $\psi\left(x^{\prime}\right)$ is found as

$$
\psi(x)=(2 \pi)^{-1 / 2} \int \tilde{F}(y) / \tilde{K}(y) \times \exp (-i x y) \mathrm{dy},
$$

where $\widetilde{F}(y)$ is the Fourier transform of the function $F(x)$ described by

$$
\tilde{F}(y)=(2 \pi)^{-1 / 2} \int F(x) \exp (-i y x) d y,
$$

and $\widetilde{K}(y)$ is the Fourier transform of the function $K\left(x-x^{\prime}\right)$.

The method of increasing the resolution as opposed to other methods does not require any preliminary assumptions as to the number of peaks (components), their intensities, and positions in spectrum and does not generate additional artefacts. The following pulse wave parameters were recorded: pulse, saturation, ventricle/ aorta volume ratio, aorta valve, pulsatility index, $k 1 / k 2$ index, and artery dynamic. The correlations between the total dose adminis-

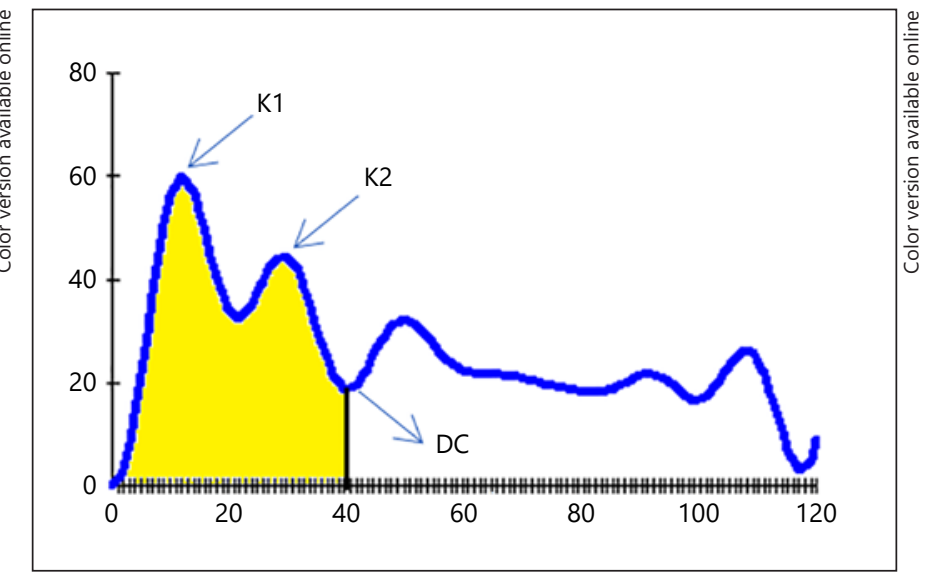

Fig. 2. The results of signal resolution for individual evolution of the pulse wave. $k 1$ and $k 2$, characteristic points correlated with the expansion of the aorta; DC, characteristic point correlated with aortic valve closure.

tered to the target $D_{c}$, the mean heart dose $D_{s}$, and the maximum dose to the coronary arteries $D_{t}$, as well as the treatment duration $t$, and changes in individual parameters determined with HSR pulse oximetry were analyzed. Changes in pulse oximetry-based parameters (pulse wave) were each time calculated according to the following equation:

$$
\Delta x=x_{2}-x_{1},
$$

where $x_{1}$ is the value of the tested pulse wave parameter before administering a specific dose and $x_{2}$ is the value of the tested pulse wave parameter after administering a specific dose.

The Shapiro-Wilk test was used to evaluate data distribution. If data followed a normal distribution, the Pearson correlation coefficient was used to investigate the correlations; otherwise, the Spearman's correlation coefficient was applied (Statistica 13). Correlations were considered statistically significant at $p<0.05$.

\section{Results}

Figure 1 shows an example screenshot with the HSR pulse oximetry results, which are presented in a comprehensible way to the patient (i.e., in a bar chart). The pulse wave recording and its Fourier transform are demonstrated, as well as the age-specific reference ranges. Table 1 shows the statistical analysis of the recorded parameters. Tables $2-5$ present the correlation analysis between the following variables: the dose administered to the target $D_{c}$, the mean heart dose $D_{s}$, and the maximum dose to the coronary arteries $D_{t}$ (in the analyzed period), as well as treatment duration $t$, and changes in individual parameters determined with HSR pulse oximetry. 
Table 1. Statistical data on the pulse wave parameters

\begin{tabular}{llllllll}
\hline Pulse wave parameter & Mean & Min & Max & SE & Median & 25th percentile & 75th percentile \\
\hline Dc, Gy & 57.70 & 17.50 & 102.00 & 5.13 & 60.75 & 40.50 & 70.00 \\
$D$, Gy & 6.24 & 0.77 & 25.96 & 1.66 & 3.02 & 1.64 & 7.15 \\
$D t$, Gy & 6.80 & 0.00 & 14.05 & 0.99 & 6.39 & 3.56 & 10.20 \\
$t$, days & 26.26 & 7.00 & 51.00 & 2.53 & 25.00 & 18.00 & 33.00 \\
$\Delta$ pulse, 1/min & 1.97 & -12.00 & 27.00 & 2.25 & -0.500 & -5.00 & 10.00 \\
$\Delta$ saturation (\%) & 0.00 & -4.00 & 3.00 & 0.40 & 0.00 & -1.00 & 1.00 \\
$\Delta$ ventricle/aorta volume ratio & 0.47 & -23.80 & 42.89 & 2.79 & -0.29 & -3.10 & 1.79 \\
$\Delta$ aorta valve & 0.13 & -1.60 & 3.79 & 0.33 & 0.00 & -1.20 & 1.09 \\
$\Delta$ pulsatility index & 0.00 & -0.89 & 0.49 & 0.08 & 0.09 & -0.21 & 0.21 \\
$\Delta$ k1/k2 index & 0.16 & -0.39 & 0.59 & 0.06 & 0.14 & 0.05 & 0.38 \\
$\Delta$ artery dynamic & -2.71 & -26.00 & 50.00 & 4.24 & -8.99 & -15.01 & 5.01 \\
\hline
\end{tabular}

Data are presented as median and interquartile range because not all data follow a normal distribution. $\mathrm{SE}$, standard error; $D c$, the dose administered to the target; $D s$, the mean heart dose; $D t$, the maximum dose to the coronary arteries, given in the analyzed treatment duration $t$.

Table 2. Correlation between the total dose administered and prescribed to the target $D c$ and changes in individual parameters determined with HSR pulse oximetry in the analyzed period

\begin{tabular}{lll}
\hline Correlation between & $\begin{array}{l}\text { Correlation } \\
\text { coefficient }\end{array}$ & $p$ value \\
\hline$\Delta$ pulse and $D c^{1}$ & 0.09 & 0.70 \\
$\Delta$ saturation and $D c^{1}$ & 0.05 & 0.84 \\
$\Delta$ ventricle/aorta volume ratio and $D c^{2}$ & 0.50 & 0.03 \\
$\Delta$ aorta valve and $D c^{1}$ & 0.43 & 0.06 \\
$\Delta$ pulsatility index and $D c^{1}$ & -0.30 & 0.22 \\
$\Delta k 1 / k 2$ index and $D c^{1}$ & -0.16 & 0.51 \\
$\Delta$ arterial dynamics and $D c^{2}$ & -0.16 & 0.51 \\
\hline
\end{tabular}

HSR, high signal resolution. ${ }^{1}$ Pearson's correlation. ${ }^{2}$ Spearman's correlation.

Table 4. Correlation between the maximum dose to the coronary arteries $D t$ and changes in individual parameters determined with HSR pulse oximetry

\begin{tabular}{lll}
\hline Correlation between & $\begin{array}{l}\text { Correlation } \\
\text { coefficient }\end{array}$ & $p$ value \\
\hline$\Delta$ pulse and $D t^{1}$ & 0.18 & 0.54 \\
$\Delta$ saturation and $D t^{1}$ & -0.24 & 0.41 \\
$\Delta$ ventricle/aorta volume ratio and $D t^{2}$ & -0.45 & 0.11 \\
$\Delta$ aorta valve and $D t^{1}$ & -0.28 & 0.33 \\
$\Delta$ pulsatility index and $D t^{1}$ & 0.26 & 0.38 \\
$\Delta k 1 / k 2$ index and $D t^{1}$ & 0.50 & 0.07 \\
$\Delta$ artery dynamic and $D t^{2}$ & 0.13 & 0.65 \\
\hline
\end{tabular}

HSR, high signal resolution. ${ }^{1}$ Pearson's correlation. ${ }^{2}$ Spearman's correlation.
Table 3. Correlation between the mean heart dose $D$ s and changes in individual parameters determined with HSR pulse oximetry

\begin{tabular}{lll}
\hline & $\begin{array}{l}\text { Correlation } \\
\text { coefficient }\end{array}$ & $p$ value \\
\hline$\Delta$ pulse and $D s^{2}$ & 0.25 & 0.31 \\
$\Delta$ saturation and $D s^{2}$ & 0.10 & 0.69 \\
$\Delta$ ventricle/aorta volume ratio and $D s^{2}$ & 0.19 & 0.45 \\
$\Delta$ aorta valve and $D s^{2}$ & -0.14 & 0.57 \\
$\Delta$ pulsatility index and $D s^{2}$ & 0.09 & 0.72 \\
$\Delta k 1 / k 2$ index and $D s^{2}$ & 0.00 & 1.00 \\
$\Delta$ artery dynamic and $D s^{2}$ & 0.07 & 0.78 \\
\hline
\end{tabular}

HSR, high signal resolution. 'Pearson's correlation. ${ }^{2}$ Spearman's correlation.

Table 5. Correlation between the treatment duration $t$ and changes in individual parameters determined with HSR pulse oximetry

\begin{tabular}{lll}
\hline Correlation between & $\begin{array}{l}\text { Correlation } \\
\text { coefficient }\end{array}$ & $p$ value \\
\hline$\Delta$ pulse and $t^{1}$ & 0.13 & 0.60 \\
$\Delta$ saturation and $t^{1}$ & 0.07 & 0.77 \\
$\Delta$ ventricle/aorta volume ratio and $t^{2}$ & 0.48 & 0.04 \\
$\Delta$ aorta valve and $t^{1}$ & 0.38 & 0.11 \\
$\Delta$ pulsatility index and $t^{1}$ & -0.26 & 0.28 \\
$\Delta k 1 / k 2$ index and $t^{1}$ & -0.16 & 0.51 \\
$\Delta$ artery dynamic and $t^{2}$ & -0.10 & 0.68 \\
\hline
\end{tabular}

HSR, high signal resolution. 'Pearson's correlation. ${ }^{2}$ Spearman's correlation.
86

Pulse 2021;9:83-88

DOI: $10.1159 / 000519100$
Jezierska et al. 


\section{Discussion/Conclusion}

We found the dose administered to the target $D_{c}$ and the treatment duration $t$ both significantly positively correlated with the change in ventricle/aorta volume ratio parameter. This finding suggests that the change in ventricle/aorta volume ratio is larger as the dose $D_{c}$ increases, and thereby the treatment duration $t$. The strength of the relationship can be considered moderate (significant correlation).

The ventricle/aorta volume ratio parameter is the ratio between the area under the HSR-PW generated by the contraction of the left ventricle until the aortic valve closes and the area of the HSR-PW responsible for expansion and contraction of the aorta. Therefore, this parameter reflects the fact that the aorta is susceptible to changes in blood pressure caused by left ventricular contraction [16].

This pilot study involved only a small group of patients, with the primary aim of identifying pulse wave parameters that correlate with the dose delivered to the patient during radiotherapy. Therefore, our findings require further confirmation in a larger cohort of patients. From a clinical point of view, it will be most important to verify the correlation between the change in ventricle/ aorta volume ratio parameter and observed cardiovascular complications of radiotherapy, which was not examined in the current study. Founded correlation could be real or could be a type I error more frequently associated with a small, noncontrolled study design. In addition, further monitoring of patients using HSR pulse oximetry over a longer period of time, in association with any treatment-related side effects, would help us determine whether this method could be used to predict serious and late cardiovascular complications. Correlations between the pulse wave parameters and the other treatment plan parameters that are not acceptance criteria (such as maximum dose to the heart or aorta) should also be investigated.
In conclusion, our preliminary analysis indicates that the HSR pulse oximetry test, especially the ventricle/aorta volume ratio parameter, is a potential prognostic indicator of toxicity from radiation for breast and lung cancers. This method has several advantages, as the HSR pulse oximeter is relatively inexpensive, and the test itself is quick and noninvasive. In addition, the use of HSR pulse oximetry is amenable to telemonitoring and the long-term self-monitoring of cardiovascular health, whereby patients would only be required to visit a doctor if they developed more serious conditions.

\section{Statement of Ethics}

In accordance with the decision KB-0012/19/16 issued by the Pomeranian Medical University in Szczecin on March 13, 2016, all patients eligible for radiotherapy were offered participation in the study after being informed about the purpose of the research and its process. All participants provided written informed consent to participate in the study.

\section{Conflict of Interest Statement}

The authors have no conflicts of interest to declare.

\section{Funding Sources}

The authors received no financial support for the research, authorship, and publication of this article.

\section{Author Contributions}

K.J. conceived and designed research. K.J., A.M., P.W., and M.Ł. conducted experiments. K.J. and R.K. analyzed data. K.J., A.S.N., P.W., and W.P. wrote the manuscript. All authors read and approved the manuscript.

\section{Data Availability Statement}

The research data are not publicly available on ethical grounds.

\section{References}

1 Howlader N, Noone A, Krapcho M, Miller D, Bishop K, Altekruse S, et al. SEER cancer statistics review 1975-2013 National Cancer Institute; 2016.

2 Schubert LK, Gondi V, Sengbusch E, Westerly DC, Soisson ET, Paliwal BR, et al. Dosimetric comparison of left-sided whole breast irradiation with 3DCRT, forward-planned IMRT, inverse-planned IMRT, helical tomotherapy, and topotherapy. Radiother Oncol. 2011;100:241-6.

High Signal Resolution Pulse Oximetry
3 Jagsi R, Moran J, Marsh R, Masi K, Griffith KA, Pierce LJ. Evaluation of four techniques using intensity-modulated radiation therapy for comprehensive locoregional irradiation of breast cancer. Int J Radiat Oncol Biol Phys. 2010;78:1594-603.

4 Lohr F, El-Haddad M, Dobler B, Grau R, Wertz HJ, Kraus-Tiefenbacher U, et al. Potential effect of robust and simple IMRT approach for leftsided breast cancer on cardiac mortality. Int J Radiat Oncol Biol Phys. 2009;74:73-80.
5 Ares C, Khan S, MacArtain AM, Heuberger J, Goitein G, Gruber G, et al. Postoperative proton radiotherapy for localized and locoregional breast cancer: potential for clinically relevant improvements? Int J Radiat Oncol Biol Phys. 2010;76:685-97.

6 Gulybán A, Kovacs P, Sebestyen Z, Farkas R, Csere T, Karácsonyi G, et al. Multisegmented tangential brest fields: a rational way to treat breast cancer. Strahlenther Onkol. 2008;184: 262-9. 
7 Aznar MC, Korreman SS, Pedersen AN, Persson GF, Josipovic M, Specht L. Evaluation of dose to cardiac structures during breast irradiation. Br J Radiol. 2007;84:743-6.

8 Taylor CW, Povall JM, McGale P, Nisbet A, Dodwell D, Smith JT, et al. Cardiac dose from tangential breast cancer radiotherapy in the year 2006. Int J Radiat Oncol Biol Phys. 2008; 72:501-7.

9 Darby SC, Cutter DJ, Boerma M, Constine LS, Fajardo LF, Kodama K, et al. Radiation-related heart disease: current knowledge and future prospects. Int J Radiat Oncol Biol Phys. 2010;76(3):656-65.
10 Darby SC, Ewertz M, McGale P, Bennet AM, Blom-Goldman U, Brønnum D, et al. Risk of ischemic heart disease in women after radiotherapy for breast cancer. N Engl J Med. 2013; 368:987-98.

11 Kamat V. Pulse oximetry. Indian J Anaesth. 2002;46(4):261-8.

12 Krzyminiewski R, Panek G, Stepień R. High resolution vectorcardiogram. J Med Phys. 1999;24:181-5.

13 Krzyminiewski R. High signal resolution pulsoximeter in home care telemonitoring. Glob Telemed eHealth Updates. 2010;3:217-21.
14 Szymił A, Krzyminiewski R, Dobosz B, Pająk A, Szyszka A, Ładzińska M. Pulse wave shape analysis of the cardiovascular system using high signal resolution. Curr Top Biophys. 2014;37:55-62.

15 Krzyminiewski R, Dobosz B, Ładzinska M, Jemielity M, Buczkowski P, Urbanowicz T, et al. High signal resolution pulse wave - hope for a fast and cheap home care monitoring patients with cardiac diseases. Med-e-Tel Conf Proc. 2011;4:540-4.

16 Krzyminiewski R, Mrozińska G, Dobosz B. The impact of fruit-vegetable diet on high signal resolution pulse wave $(\mathrm{Hsr}-\mathrm{Pw})$ parameters. J Nutr Health Aging. 2017;22(3):420-4. 